\title{
Usabilidade Corporativa: Análise do Impacto na Produtividade dos Negócios
}

\author{
Rodrigo Costa dos Santos ${ }^{1}$, Maria Augusta S. Machado ${ }^{2}$, Marcelo Guedes Carneiro ${ }^{3}$ \\ ${ }^{1,2}$ Faculdades IBMEC-RJ, Av. Pres. Wilson, 118 - Centro, Rio de Janeiro, RJ. \\ ${ }^{3}$ ESPM - Escola Superior de Propaganda e Marketing - Av. Rio Branco, 53, Centro- RJ. \\ ${ }^{1}$ rodrigo.santos@al.ibmecrj.edu.br ; ${ }^{2}$ mmachado@ibmecrj.br ; ${ }^{3}$ marguedes@ terra.com.br
}

\begin{abstract}
Resumo
As organizações hoje cada vez mais utilizam e dependem da tecnologia da informação. Os sistemas corporativos estão cada vez mais modernos e exigem um alto potencial de manuseio por parte dos seus usuários. Neste sentido, a usabilidade de sistemas é fundamental, pois é a característica de determinado software ser fácil de usar e de aprender, ser eficiente, eficaz, provocar poucos erros e proporcionar satisfação aos seus usuários. Porém, nem sempre essas características são levadas em consideração na construção de interfaces de sistemas como meio para se conseguir vantagens competitivas, ou no aumento da produtividade da empresa. Para validar o estudo foi feita uma análise de uma ferramenta de software de uma grande empresa do ponto de vista da usabilidade. Escolheu-se a intranet por se tratar de uma das ferramentas mais utilizada pelos funcionários, seja ele do nível operacional ou gerencial. Foram aplicados questionários a fim de medir vários construtos da usabilidade e depois avaliados sob duas metodologias: estatística convencional e outra usando matemática nebulosa. Verificou-se que não existe um valor final para a usabilidade, mas sim várias avaliações por cada métrica utilizada. Outra constatação foi que com simples mudanças, pode-se aumentar a usabilidade e por conseqüência a produtividade dos funcionários. Palavras-chave: Usabilidade Corporativa; Produtividade; Matemática Nebulosa.
\end{abstract}

\begin{abstract}
Nowadays the organizations use and depend on information technology. The corporative systems are more and more modern and demand a high potential of handling for part of their users. In this sense, systems usability is basic. Systems usability is the characteristic of determined software to be easy of using and of learning, being efficient, provoking few errors and providing satisfaction to users. However, not always these characteristics are taken into account in the construction of system's interfaces in order that competitive advantages are got, or in the increase of the enterprise's productivity. To validate the study there was done an analysis of a great enterprise's software on the point of view of the usability. The intranet was chosen because it is one of the tools most used by operational or management employees. Questionnaires were applied in order to measure several usability constructs and then valued under two methodologies: one of the conventional statistic and the other using fuzzy logic. It was seen that does not exist a final value for usability, but several evaluations for each used metric. Another observation was that with simple changes, it is possible to increase the usability and consequently the productivity of the employees.
\end{abstract}

Key-words: Corporative Usability; Productivity; Fuzzy Logic.

\section{Introdução}

Com a grande competitividade, as empresas vêm se reestruturando sensivelmente em todas as suas áreas para a conquista de novas fatias de mercado, pois a chamada a novos clientes e a manutenção dos existentes, dependerá sempre dos seus bons serviços prestados e/ou do seu histórico. Nessa busca exacerbada por crescimento, as empresas correm riscos elevados, acarretando a necessidade de manter seus produtos com alto padrão de qualidade demandado pelos clientes cada vez mais exigentes.
Essa necessidade também reflete na qualidade da informação e torna as ferramentas de software uma parte fundamental dos sistemas de informação. As ferramentas de software constituem um grande veículo de produção para todos os tipos de organizações e compreende várias finalidades dentro da empresa: controles, finanças, contabilidade, estratégias, vendas, colaborações eletrônicas, troca de mensagens, etc.

Todavia, mesmo com as vantagens já conhecidas advindas da tecnologia da informação, devem ser estudadas e consideradas suas implicações, limitações, possibilidades e especificidades. 
O impacto do computador na sociedade é iminente. É sabido que nos últimos vinte anos, o computador cada vez mais se torna parte integrante do dia-a-dia das pessoas. Mais e mais dispositivos "computadorizados" surgem diariamente. É só lembrar como eram certos aparelhos, veículos, telefones e até mesmo o próprio computador há alguns anos atrás.

$\mathrm{O}$ presente artigo pretende avaliar uma das ferramentas de software existente em uma empresa, que é a intranet, sob o ponto de vista da usabilidade e verificar como a usabilidade pode tornar o trabalho dos funcionários mais produtivos com o uso de uma intranet com um alto grau de usabilidade.

Para validar a pesquisa, foi avaliada a usabilidade da intranet de uma grande empresa, ou seja, sua facilidade de uso, eficiência e eficácia na realização de suas tarefas e na satisfação final que o sistema proporciona aos seus usuários. Os dados foram coletados através de questionários e os resultados obtidos através de dois métodos: a estatística convencional e de uma metodologia usando a matemática nebulosa.

Primeiramente é realizada uma revisão da literatura com relação à usabilidade de sistemas, intranet nas organizações e sobre a matemática nebulosa. Depois é apresentada a metodologia utilizada para realização da pesquisa, seguida da avaliação dos resultados. Finalizando com as conclusões da pesquisa.

Verificou-se que não existe um resultado único para avaliar o grau de usabilidade de um sistema, mas sim um conjunto de critérios ou métricas que são selecionados para a avaliação da usabilidade, que podem apresentar resultados diferentes entre si.

\section{Revisão da Literatura}

\subsection{Usabilidade de Sistemas}

O termo usabilidade é freqüentemente usado para se referir à capacidade de um produto ser facilmente usado; porém, somente essa definição simplifica demais o problema, que envolve atender as expectativas do usuário de forma completa.

A usabilidade de sistemas especificamente tem como objetivo elaborar interfaces de sistemas capazes de permitir uma interação fácil, agradável, com eficácia e eficiência. Ela deve capacitar a criação de interfaces transparentes de maneira a não dificultar o seu uso, permitindo ao usuário pleno controle do ambiente sem se tornar um obstáculo durante a interação.

Quando um sistema possui uma interface amigável e possibilita ao usuário utilizá-lo de forma intuitiva, a usabilidade pode se tornar um fator de motivação e ter seu usuário como um aliado, ao passo que se essa motivação não for atingida, essa situação pode-se reverter e se tornar um fator de rejeição do sistema [14].

A usabilidade pode ser medida sob vários aspectos. Segundo a ISO 9241 [8], por exemplo, a usabilidade de sistemas pode ser medida através de indicadores de eficiência, eficácia e satisfação dos seus usuários.

Com relação às técnicas para avaliação de usabilidade de sistemas, existem várias: baseadas em questionários aplicados aos usuários, baseadas em modelos formais, base de conhecimento, checklists, ensaios de interação ou sistemas de monitoramento [4].

No caso de um software que já existente ou que está sendo utilizado em uma empresa, a técnica mais aderente é a aplicação de questionário, pois ninguém melhor do que os próprios usuários para opinarem a respeito da usabilidade do sistema. Eles que estão em contato com o sistema no dia-a-dia.

\subsection{Intranet nas Organizações}

Com inovação da tecnologia da informação e comunicação (TIC), o sucesso das empresas passou a depender diretamente da sua habilidade em inovar serviços, produtos e processos.Por isso, a TIC constitui de uma ferramenta fundamental para ajudar as empresas cumprirem essa missão.

A intranet é uma ferramenta que foi amplamente utilizada pelas organizações nesse processo de modernização. A intranet é uma rede privada que interliga os computadores dentro de uma organização, usando tecnologia da Internet, aberta somente aos seus empregados [6].

Diversas empresas já possuem sofisticadas e disseminadas redes de intranet. As intranets podem ser úteis em uma variedade de funções empresariais: inteligência de negócios, serviços públicos, informações corporativas e atendimento ao cliente [17].

$\mathrm{O}$ retorno de investimento em projetos de intranet tem sido bem acima do esperado por parte das organizações. Com o uso de intranets, as empresas buscam criar uma ligação mais robusta e uniforme entre os recursos de dados espalhados pela organização. Trocar soluções departamentais, em que dados e relatórios são desenvolvidos para pequenas comunidades especializadas de usuários, por uma intranet empresarial, coloca os recursos de dados de uma empresa em uma base mais ampla e permite que uma gama maior de usuários acesse essa informação [9].

Entre os principais benefícios obtidos com a intranet, pode-se destacar: alcance mundial, acesso de baixo custo, executável em todas as plataformas, interface-padrão única, criação padronizada de documentos, redução de custos com papel, redução de custos com marketing e vendas, aumento da produtividade via acesso mais rápido a informações e colaboração mais fácil [12].

De qualquer forma, diversos desafios precisam ser trabalhados de forma a ampliar e aprimorar o uso da intranet. Segundo [12], destacam-se as seguintes limitações de projetos de intranet: falta de administração de desempenho, apoio mínimo ao usuário, dificuldade de manutenção do conteúdo ao longo do tempo, aplicativos lentos, informações não filtradas 
sobrecarregando os usuários e a falta de acesso à rede para determinados funcionários.

Além dessas limitações, a intranet precisa ser desenhada de forma que seja de fácil usabilidade permitindo, dessa forma, que os usuários não criem resistência para adotá-la e que o índice de erros seja o menor possível, promovendo dessa forma a tão esperada produtividade por parte da empresa promotora do projeto.

\subsection{Matemática Nebulosa}

A matemática nebulosa ou difusa foi criada em 1965 a partir da publicação do artigo intitulado "Fuzzy Sets" por Lofti A. Zadeh, engenheiro eletrônico e professor da Universidade da Berkeley, Califórnia [2].

Os Conjuntos Nebulosos e a Lógica Nebulosa fornecem a base para geração de técnicas poderosas para a solução de problemas, com uma vasta aplicabilidade, especialmente nas áreas de Engenharia de Controle e tomada de decisões.

A força da Lógica Nebulosa deriva da sua habilidade em inferir conclusões e gerar respostas baseadas em informações vagas, ambíguas, incompletas e imprecisas. Neste aspecto, os sistemas Nebulosos têm a habilidade de raciocinar de forma semelhante a dos humanos. Seu comportamento é representado de maneira muito simples e natural, levando à construção de sistemas compreensíveis e de fácil manutenção [1].

A teoria dos Conjuntos Nebulosos é uma generalização da teoria dos Conjuntos Tradicionais para resolver os paradoxos gerados a partir da classificação "verdadeiro ou falso" da Lógica Clássica. Tradicionalmente, uma proposição lógica tem dois extremos: ou "completamente verdadeiro" ou "completamente falso". Entretanto, na Lógica Nebulosa, uma premissa varia em grau de verdade de 0 a 1 , o que leva a ser parcialmente verdadeira ou parcialmente falsa. Com a incorporação do conceito de "grau de verdade", a teoria dos Conjuntos Nebulosos estende a teoria dos Conjuntos Tradicionais. Os grupos são rotulados qualitativamente (usando termos lingüísticos, tais como: alto, morno, ativo, pequeno, perto, etc.) e os elementos destes conjuntos são caracterizados variando o grau de pertinência (valor que indica o grau em que um elemento pertence a um conjunto). Por exemplo, temperaturas entre $30^{\circ}$ (trinta graus) e $40^{\circ}$ (quarenta graus) pertencem ao conjunto das "temperaturas altas", embora a temperatura de $40^{\circ}$ tenha um grau de pertinência maior neste conjunto [13].

De maneira não muito bem compreendida, humanos têm a capacidade de associar um grau de pertinência a um determinado objeto sem compreender conscientemente como se chega a ele. Por exemplo, um aluno não teria dificuldade em assinalar um grau ao professor no conjunto dos "bons professores". Esse grau é alcançado imediatamente sem nenhuma análise consciente sobre os fatores que influem nessa decisão [3].
Essa técnica da lógica nebulosa foi empregada na presente pesquisa para elaboração do questionário e no processamento dos resultados após a coleta dos dados, tendo em vista ser uma técnica que consegue unir a linguagem humana da matemática.

\section{$3 \quad$ Metodologia}

O presente estudo apresenta características de pesquisa aplicada, pois tem por objetivo utilizar um caso real para fundamentar sua análise. A pesquisa aplicada é motivada pela necessidade de se resolver problemas concretos com finalidade prática [18].

Inicialmente, foi escolhida para ser analisada a intranet das Centrais Elétricas Brasileiras S/A Eletrobrás.

A Eletrobrás foi criada em 1962 pelo governo brasileiro para promover estudos e projetos de construção e operação de usinas geradoras, linhas de transmissão e subestações. Com o passar do tempo, ela adquiriu características de holding, controlando empresas de geração e transmissão de energia elétrica em todo país.

Atualmente, a Eletrobrás é uma empresa de economia mista e de capital aberto, com ações negociadas nas Bolsas de Valores de São Paulo, Madri e Nova Iorque. O Governo Federal possui mais da metade das ações $(52,45 \%)$. As empresas do Grupo Eletrobrás produzem cerca de $60 \%$ da energia elétrica consumida no país [5].

A Intranet da Eletrobrás é o principal canal de comunicação da empresa com seus funcionários, além de hospedar os principais serviços realizados por eles no dia-a-dia, como consultas relativas a Recursos Humanos, contracheque, freqüência eletrônica, solicitação de viagens, reembolso de táxi, abertura de chamados para help-desk de informática e para manutenção predial, clipping de notícias relacionadas ao setor elétrico, avisos e notícias corporativas, consulta ao acervo da biblioteca, lista telefônica dos funcionários, etc.

Antes de começar a análise da intranet do ponto de vista da usabilidade, conforme especificado na ISO 9241 [8], deve-se identificar primeiro o contexto de uso sob quatro aspectos: Usuários, Tarefas, Equipamentos e Ambientes. Essa especificação consiste em descrever, de forma sucinta, cada um dos aspectos de modo que se possa identificar cada contexto em que o sistema será analisado. Assim foi feito para intranet da Eletrobrás:

\section{a) Especificação dos Usuários}

Os usuários alvos desta pesquisa são funcionários da empresa com tempo de casa variando entre 3 a 20 anos, de ambos os sexos, todos com nível superior, com experiência no uso de computador e grande experiência no uso da intranet da empresa, visto que a utilizam diariamente (de segunda a sexta-feira) para realizar tarefas do cotidiano do trabalho. 


\section{b) Especificação das Tarefas}

A intranet foi avaliada como um todo, porém foi dado destaque nas tarefas abaixo:

-

$\bullet$

- $\quad$ Sistema de solicitação de viagens; e

- Lista telefônica dos funcionários.

\section{c) Especificação dos Equipamentos}

Os usuários utilizam a intranet através de suas estações de trabalho. As estações de trabalho da Eletrobrás possuem configuração semelhante, podendo apresentar pequenas variações com relação à memória RAM; porém, em geral são máquinas com processadores entre 2,5 a $3 \mathrm{Ghz}$, com $256 \mathrm{MB}$ de memória RAM, possuindo sistema operacional Windows XP Professional com o navegador Internet Explorer versão 6.0, constituindo assim um ambiente homogêneo para todos os usuários.

\section{d) Especificação do Ambiente}

Os funcionários da Eletrobrás trabalham sete horas e meia por dia, com uma hora e meia de almoço. As instalações da empresa são boas, possuindo toda infraestrutura necessária. Os postos de trabalho possuem boa ergonomia para os funcionários, com boa iluminação e temperatura regulada por um ar-condicionado central. $\mathrm{O}$ único inconveniente do ambiente é com relação à acústica, pois os setores são separados por divisórias baixas e se consegue ouvir conversas paralelas e ruídos indesejados.

Os funcionários costumam ser avaliados por competências e resultados e a cobrança do chefe no diaa-dia costuma ser moderada.

Uma vez especificado o contexto de uso dos usuários da intranet da empresa, e por consequiência conhecido o público-alvo da pesquisa, foram selecionados trinta funcionários com o perfil indicado na descrição dos usuários para constituírem a amostra desta avaliação. Foi aplicado um questionário para avaliar a usabilidade da intranet a esses trinta funcionários.

Os questionários foram aplicados entre os dias 06/11/2006 e 14/11/2006 no horário do trabalho. Dos trinta questionários, foram descartados três; pois, apresentaram algumas inconsistências como questões em branco, por exemplo, restando 27 questionários.

As opiniões dos usuários foram tabuladas em planilha eletrônica. O objetivo da planilha eletrônica foi executar os seguintes passos: consolidar as opiniões dos usuários e determinar a freqüência das opiniões dos usuários.

Para avaliar a usabilidade, primeiramente recorreu-se à ISO (International Organization for Standardization), que determina que a usabilidade é um dos itens considerados no tratamento de qualidade de software, através das suas normas 9126 e também pela norma 9241.

Porém, as normas da ISO não trazem um conjunto de critérios ou métricas para avaliação de usabilidade de sistemas. Por isso, as métricas utilizadas nesse estudo serão aquelas apresentadas por [16], que estipulou um conjunto de métricas para avaliação de usabilidade de sistema baseado numa revisão da literatura de bases científicas brasileiras entre 1995 a 2006. Essas métricas de usabilidade de sistemas estão baseadas na ISO 9126 e nos critérios de avaliação segundo alguns autores como Shackel, Nielsen, Bastien \& Scapin, Jordan, Shneiderman e Quesenbery [16].

Segundo [16], as métricas consideradas para avaliação de usabilidade, e as que serão utilizadas por esta pesquisa são:

- $\quad$ Facilidade de aprender

- Facilidade de relembrar

- Controle de erros

- Eficiência

- $\quad$ Eficácia

- $\quad$ Satisfação

A facilidade de aprender ou inteligibilidade, segundo a ISO 9126 [7], é a capacidade de o software possibilitar ao usuário aprender a manuseá-lo.

Essa métrica está sendo avaliada pelos seguintes construtos:

A) Facilidade que o usuário tem ao completar uma tarefa com sucesso pela primeira vez;

B) Primeira impressão que o usuário tem ao utilizar o sistema;

C) Número de tentativas realizadas para aprender a concluir uma tarefa;

D) Tempo para conseguir aprender a realizar uma tarefa com sucesso;

E) Facilidade de aprender uma tarefa;

F) Número de possibilidades diferentes que o sistema oferece para realizar a mesma tarefa, por exemplo: caminho padrão versus teclas de atalhos, caminhos mais curtos, macros, botões específicos, etc;

G) Ganho de produtividade com relação à maneira mais rápida que o usuário consegue realizar uma tarefa, comparando com a maneira padrão que o sistema oferece por padrão;

H) Flexibilidade que o sistema tem para executar as tarefas de maneiras diferentes, como por exemplo: personalização de atalhos, valores, menus, macros, etc;

I) Capacidade de o sistema guiar através de sua execução com dicas, ajuda, avisos, etc;

J) Rapidez para completar uma tarefa com sucesso no sistema pela primeira vez.

A facilidade de relembrar, segundo Nielsen [11], avalia as funcionalidades do sistema para que sejam fáceis de relembrar, mesmo após o usuário ficar certo período de tempo sem usá-lo, sem necessidade de um novo treinamento.

Essa métrica está sendo avaliada pelos seguintes construtos:

A) Relembrar como executar uma tarefa após um período de tempo sem utilizar o sistema;

B) Facilidades para relembrar a utilização do sistema;

C) Agilidade para relembrar o uso do sistema após um período de tempo sem utilizar o mesmo. 
O controle de erros, ou operacionalidade, segundo a ISO 9126 [7], é a capacidade de o software possibilitar ao usuário operá-lo e controlá-lo.

Essa métrica está sendo avaliada pelos seguintes construtos:

A) Quantidade de erros provocados pelo sistema;

B) Tempo de retomada ao funcionamento normal do sistema quando um erro ocorre;

C) Sentimento com relação à quantidade de erros provocados pelo sistema;

D) Retrabalho devido à quantidade de erros provocados pelo sistema que causa alguma perda de informação;

E) Tempo gasto para retomar a execução da tarefa no ponto em que se parou quando um erro ocorre;

F) Satisfação com relação à recuperação do erro por parte do sistema, desfazer, refazer, voltar, salvar entes de fechar, etc.;

G) Clareza das mensagens de erros apresentadas pelo sistema.

A eficiência também operacionalidade, segundo a ISO 9126 [7], é a capacidade de o software possibilitar ao usuário operá-lo e controlá-lo.

Essa métrica está sendo avaliada pelos seguintes construtos:
A) Performance apresentada pelo sistema;
B) Velocidade na realização das tarefas;
C) Produtividade do sistema;
D) Capacidade de manter o sistema sob seu controle.

A eficácia, segundo [15], avalia como as tarefas foram exatamente concluídas, e com que freqüência elas produzem erros.

Essa métrica está sendo avaliada pelos seguintes construtos:

A) Quantidade de passos para realizar uma tarefa;

B) Tempo para realizar uma tarefa qualquer no sistema;

C) Número adequado de passos para realizar uma tarefa no sistema.

A satisfação ou atratividade, segundo a ISO 9126 [7], é a capacidade do software em atrair o usuário, ser agradável.

Essa métrica está sendo avaliada pelos seguintes construtos:

A) Interação com a interface do sistema;

B) Realização das tarefas no sistema, com relação à clareza das mensagens, recuperação de erros, etc.;

C) Sentimento do usuário ao usar o sistema de maneira geral.

Após a coleta de dados e a consolidação das opiniões dos usuários, foi aplicada uma metodologia nebulosa, a fim de encontrar um número triangular nebuloso resultante das frequiências das opiniões dos usuários para o conjunto dos construtos que compõem a métrica avaliada.

Os números triangulares nebulosos são números nebulosos especiais, que apresentam duas características muito importantes: MODA e AMPLITUDE. A moda representa o valor do número nebuloso cuja pertinência é igual a 1 (um), ou seja, um ponto de convergência para as opiniões dos usuários. A amplitude, por sua vez, é a metade da base do número nebuloso e representa o intervalo de confiança do número. A amplitude é inversamente proporcional à confiança que se tem no valor da função de pertinência: quanto menor a amplitude, maior a confiança nos dados; quanto maior a amplitude, menor a confiança nos dados [2].

Com isso, como foi adotada a escala de Likert para as respostas das questões, essa escala passa a representar a MODA do número triangular nebuloso e respeita a seguinte classificação:

0-Muito Baixa;

2-Baixa;

4-Média;

6-Alta;

8-Muito Alta.

A escala de Likert é uma escala onde os respondentes são solicitados não só a concordarem ou discordarem das afirmações, mas também a informarem qual o seu grau de concordância ou discordância [10].

Com relação à AMPLITUDE, para efeito dessa pesquisa, representa a dispersão das opiniões dos usuários e é interpretada da seguinte forma:

1 - Mínima;

2 - Média;

3 - Alta;

4 - Muito Alta.

\section{$4 \quad$ Resultados}

Apresentam-se aqui as estatísticas das opiniões dos vinte e sete usuários obtidas na aplicação do questionário, bem como os números triangulares nebulosos resultantes para cada métrica e sua interpretação.

\section{1 - Descrição estatística da amostra}

Para a métrica facilidade de aprender, conforme Tabela 1 , considerando que a intranet deve ser utilizada por todos os funcionários da empresa, seja qual for o seu cargo e instrução, observa-se que sua interface apresenta uma facilidade de aprendizagem mediana.

Atenção especial deve ser dada ao construto $\mathrm{J}$, cujas respostas se concentraram em uma avaliação "baixa" de usabilidade, ou seja, quando o usuário utiliza a intranet pela primeira vez, ele demora um pouco para realizar uma tarefa com sucesso.

\begin{tabular}{|c|c|c|c|c|c|}
\hline 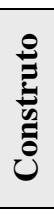 & 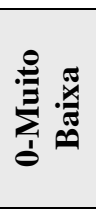 & 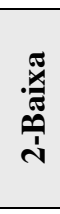 & 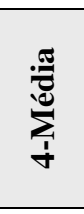 & 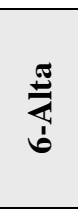 & 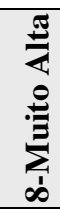 \\
\hline $\mathbf{A}$ & $0 \%$ & $0 \%$ & $37 \%$ & $56 \%$ & $7 \%$ \\
\hline B & $0 \%$ & $4 \%$ & $44 \%$ & $52 \%$ & $0 \%$ \\
\hline $\mathbf{C}$ & $0 \%$ & $4 \%$ & $19 \%$ & $74 \%$ & $4 \%$ \\
\hline
\end{tabular}




\begin{tabular}{|c|c|c|c|c|c|}
$\mathbf{D}$ & $0 \%$ & $0 \%$ & $22 \%$ & $63 \%$ & $15 \%$ \\
\hline $\mathbf{E}$ & $0 \%$ & $0 \%$ & $41 \%$ & $56 \%$ & $4 \%$ \\
\hline $\mathbf{F}$ & $0 \%$ & $4 \%$ & $30 \%$ & $59 \%$ & $7 \%$ \\
\hline $\mathbf{G}$ & $4 \%$ & $15 \%$ & $59 \%$ & $19 \%$ & $4 \%$ \\
\hline $\mathbf{H}$ & $4 \%$ & $26 \%$ & $48 \%$ & $19 \%$ & $4 \%$ \\
\hline $\mathbf{I}$ & $7 \%$ & $11 \%$ & $59 \%$ & $11 \%$ & $11 \%$ \\
\hline $\mathbf{J}$ & $11 \%$ & $41 \%$ & $37 \%$ & $11 \%$ & $0 \%$ \\
\hline
\end{tabular}

Tabela 1 - Resultados da amostra para a métrica facilidade de aprender.

Para a métrica facilidade de relembrar, conforme Tabela 2, observa-se um valor "Alta" para a facilidade de relembrar. Essa métrica apresentou um resultado satisfatório.

\begin{tabular}{|c|c|c|c|c|c|}
\hline 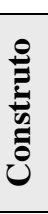 & 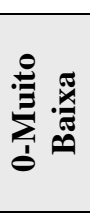 & 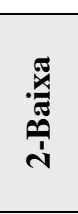 & 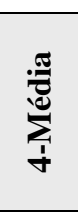 & $\begin{array}{l}\stackrel{\pi}{ٍ} \\
b\end{array}$ & 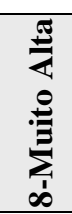 \\
\hline $\mathbf{A}$ & $0 \%$ & $4 \%$ & $30 \%$ & $52 \%$ & $15 \%$ \\
\hline B & $0 \%$ & $7 \%$ & $37 \%$ & $44 \%$ & $11 \%$ \\
\hline $\mathbf{C}$ & $0 \%$ & $15 \%$ & $22 \%$ & $52 \%$ & $11 \%$ \\
\hline
\end{tabular}

Tabela 2 - Resultados da amostra para a métrica facilidade de relembrar.

Para a métrica controle de erros, conforme Tabela 3, aparentemente, os usuários estão medianamente satisfeitos com o controle de erros apresentados pela intranet.

$\mathrm{O}$ construto $\mathrm{F}$ apresentou a pior avaliação entre as questões dessa métrica. Esse construto avalia a satisfação do usuário em relação à recuperação dos dados após a ocorrência de um erro. Aparentemente os dados são perdidos após um erro e não é apresentada para o usuário uma alternativa para se retomar o trabalho no ponto em que o erro ocorreu.

\begin{tabular}{|c|c|c|c|c|c|}
\hline 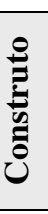 & 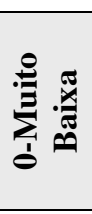 & 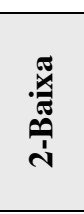 & 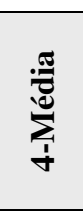 & 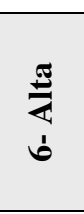 & 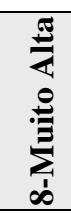 \\
\hline $\mathbf{A}$ & $0 \%$ & $0 \%$ & $22 \%$ & $56 \%$ & $22 \%$ \\
\hline B & $0 \%$ & $0 \%$ & $48 \%$ & $41 \%$ & $11 \%$ \\
\hline $\mathbf{C}$ & $0 \%$ & $22 \%$ & $19 \%$ & $48 \%$ & $11 \%$ \\
\hline D & $0 \%$ & $0 \%$ & $4 \%$ & $56 \%$ & $41 \%$ \\
\hline $\mathbf{E}$ & $0 \%$ & $7 \%$ & $33 \%$ & $52 \%$ & $7 \%$ \\
\hline $\mathbf{F}$ & $4 \%$ & $37 \%$ & $30 \%$ & $22 \%$ & $7 \%$ \\
\hline $\mathbf{G}$ & $0 \%$ & $19 \%$ & $41 \%$ & $33 \%$ & $7 \%$ \\
\hline
\end{tabular}

Tabela 3 - Resultados da amostra para a métrica controle de erros.

Para a métrica eficiência, conforme Tabela 4, aparentemente, a eficiência da intranet está “Alta”.

\begin{tabular}{|c|c|c|c|c|c|}
\hline 苞 & 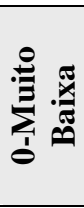 & 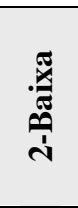 & 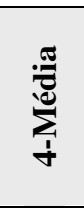 & 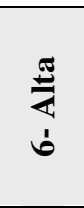 & 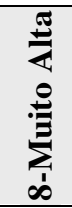 \\
\hline $\mathbf{A}$ & $0 \%$ & $11 \%$ & $37 \%$ & $44 \%$ & $7 \%$ \\
\hline B & $0 \%$ & $11 \%$ & $26 \%$ & $59 \%$ & $4 \%$ \\
\hline $\mathbf{C}$ & $0 \%$ & $7 \%$ & $30 \%$ & $52 \%$ & $11 \%$ \\
\hline D & $0 \%$ & $4 \%$ & $41 \%$ & $48 \%$ & $7 \%$ \\
\hline
\end{tabular}

Tabela 4 - Resultados da amostra para a métrica eficiência.

A eficácia da intranet, conforme Tabela 5, se apresentou de forma mediana.

\begin{tabular}{|c|c|c|c|c|c|}
\hline ن & 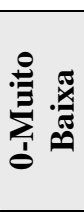 & 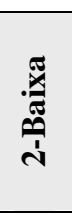 & 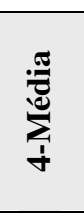 & 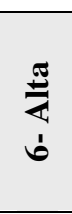 & 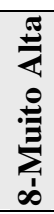 \\
\hline $\mathbf{A}$ & $0 \%$ & $7 \%$ & $56 \%$ & $37 \%$ & $0 \%$ \\
\hline B & $0 \%$ & $4 \%$ & $44 \%$ & $52 \%$ & $0 \%$ \\
\hline $\mathbf{C}$ & $0 \%$ & $19 \%$ & $41 \%$ & $37 \%$ & $4 \%$ \\
\hline
\end{tabular}

Tabela 5 - Resultados da amostra para a métrica eficácia.

Para a métrica satisfação, conforme Tabela 6 , de modo geral, os usuários estão satisfeitos. Vale ressaltar que o construto B teve a maior dispersão das opiniões dos usuários mostrando que a intranet deve melhorar a clareza das mensagens com o usuário, proporcionando maior feedback das ações tomadas pelo sistema.

\begin{tabular}{|c|c|c|c|c|c|}
\hline ن & 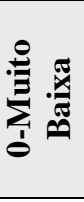 & 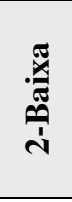 & 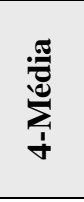 & 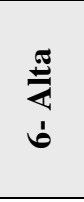 & 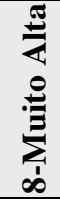 \\
\hline $\mathbf{A}$ & $0 \%$ & $11 \%$ & $19 \%$ & $63 \%$ & $7 \%$ \\
\hline B & $0 \%$ & $19 \%$ & $33 \%$ & $44 \%$ & $4 \%$ \\
\hline $\mathrm{C}$ & $0 \%$ & $4 \%$ & $19 \%$ & $74 \%$ & $4 \%$ \\
\hline
\end{tabular}

Tabela 6 - Resultados da amostra para a métrica satisfação.

\section{2 - Números Nebulosos}

Os cálculos necessários para análise Nebulosa dos resultados das métricas foram realizados utilizando o software matemático MatLab, que gerou os resultados graficamente para cada métrica estudada.

Essa análise permite se chegar a um dado mais preciso, transformando as opiniões dos usuários em termos lingüísticos inteligíveis, seguindo a escala já mencionada, ou seja, variando de "Usabilidade Muito Baixa" até "Usabilidade Muito Alta".

O gráfico apresentado para cada métrica representa dois conjuntos. $\mathrm{O}$ primeiro conjunto, simbolizado por uma linha com marcadores em forma de círculo, representa a média de todos os números nebulosos (MNN) das freqüências encontradas para as 
questões da métrica. O segundo conjunto, simbolizado pela linha com marcadores em forma de quadrado, representa o número nebuloso $(\mathrm{NN})$ na forma triangular mais semelhante ao primeiro conjunto, representando assim o resultado final para a métrica avaliada. A análise do resultado é baseada na moda e na amplitude desse número.

a) Facilidade de aprender

O número triangular nebuloso obtido para medir a facilidade de aprender está apresentado na Figura 1. Observa-se que a opinião média é 6 (usabilidade Alta) com amplitude 3 , indicando uma dispersão alta na opinião dos entrevistados. Resultado bastante impreciso, devendo-se aumentar a amostra e refazer os cálculos para se atingir maior confiabilidade no resultado.

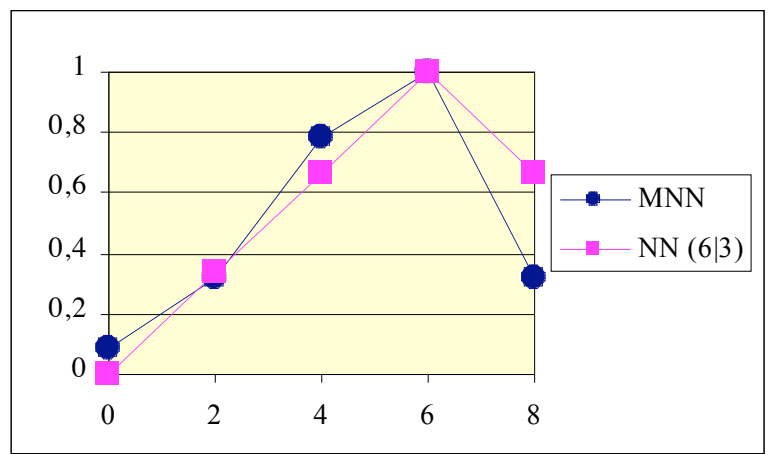

Figura 1 - Gráfico do resultado final para a métrica facilidade de aprender.

\section{b) Facilidade de relembrar}

O número triangular nebuloso obtido para medir a facilidade de aprender está apresentado na Figura 2. Observa-se que a opinião média é 6 (usabilidade Alta) com amplitude 3, indicando uma dispersão alta na opinião dos entrevistados.

Pode-se afirmar que a intranet está adequada em relação à facilidade de relembrar; porém, deve-se aumentar o tamanho da amostra com a finalidade de diminuir a amplitude do resultado.

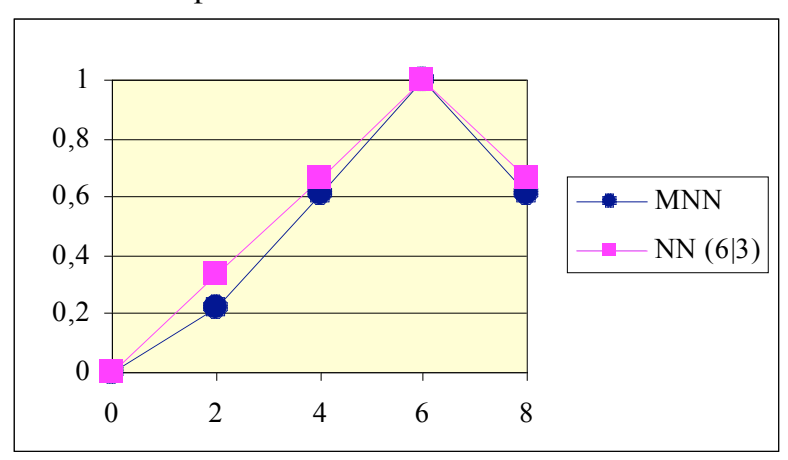

Figura 2 - Gráfico do resultado final para a métrica facilidade de relembrar.

c) Controle de Erros

O número triangular nebuloso obtido para medir o controle de erros está apresentado na Figura 3. Observase que a opinião média é 6 (usabilidade Alta) com amplitude 4, indicando uma dispersão "Muito Alta" na opinião dos entrevistados.

Pode-se afirmar que com a amplitude muito alta se tem uma total falta de confiabilidade nos dados coletados, pois houve uma dispersão muito grande nas opiniões dos usuários para essa métrica. Mesmo com a moda 6 (usabilidade Alta) nada se pode afirmar. O recomendado é aumentar a amostra ou se promover melhorias na intranet da organização com relação aos construtos relacionados ao controle de erro para então refazer a pesquisa.

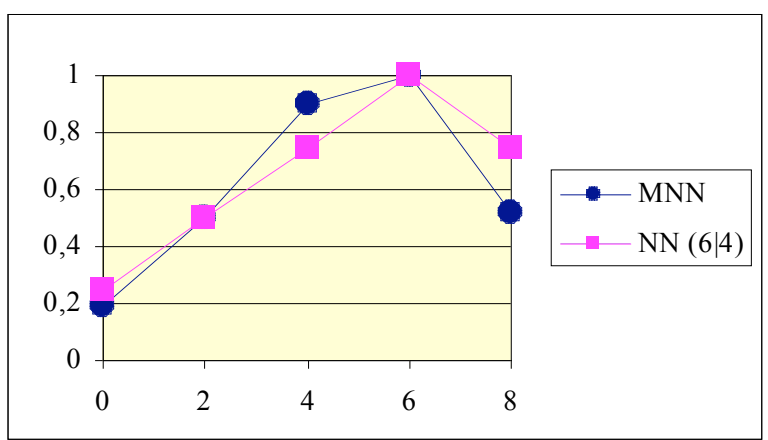

Figura 3 - Gráfico do resultado final para a métrica controle de erros.

d) Eficiência

O número triangular nebuloso obtido para medir a eficiência está apresentado na Figura 4. Observa-se que a opinião média é 6 (usabilidade Alta) com amplitude 2, indicando uma dispersão média na opinião dos entrevistados.

Pode-se afirmar que a intranet está adequada em relação à eficiência.

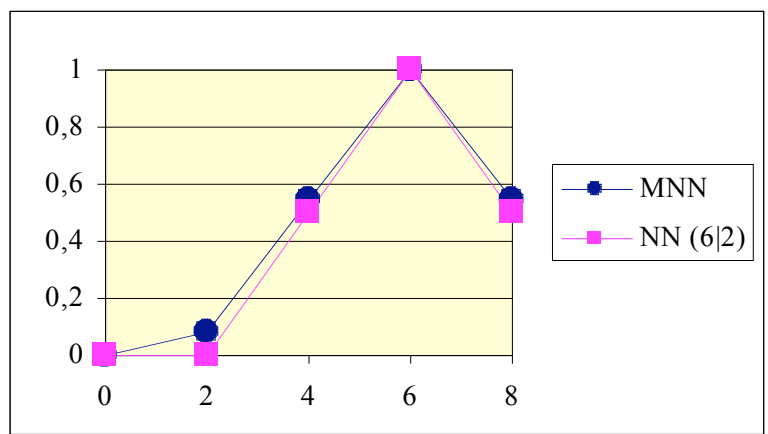

Figura 4 - Gráfico do resultado final para a métrica eficiência.

e) Eficácia

O número triangular nebuloso obtido para medir a eficácia está apresentado na Figura 5. Observa-se que a opinião média é 4 (usabilidade Média) com amplitude 2, indicando uma dispersão média na opinião dos entrevistados.

Como a dispersão é média, pode-se assumir que o resultado de que a eficácia foi avaliada medianamente pelos usuários foi aceito. 


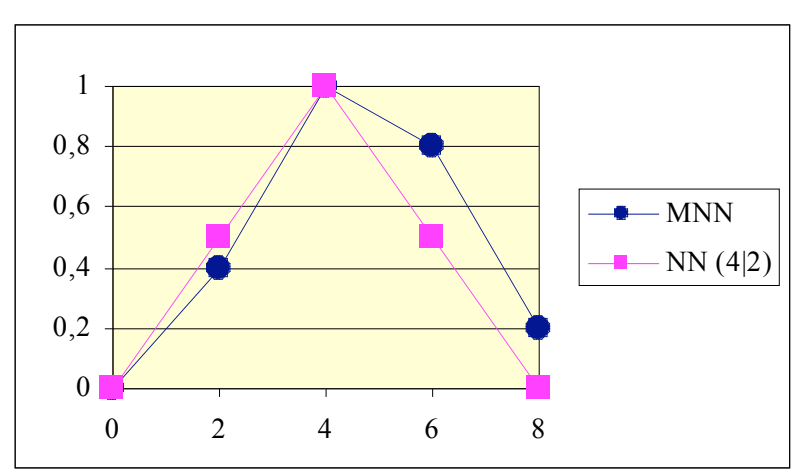

Figura 5 - Gráfico do resultado final para a métrica eficácia.

\section{f) Satisfação}

O número triangular nebuloso obtido para medir a métrica satisfação está apresentado na Figura 6 . Observa-se que a opinião média é 6 (usabilidade Alta) com amplitude 2, indicando uma dispersão média na opinião dos entrevistados. Pode-se afirmar que a satisfação geral dos usuários com a intranet da Eletrobrás é Alta.

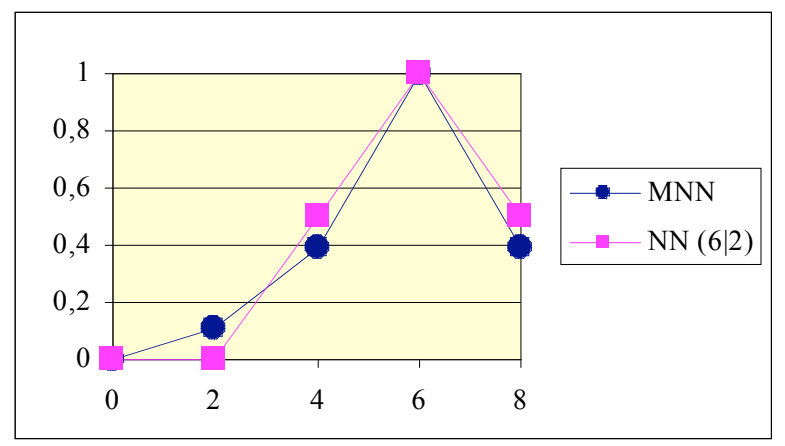

Figura 6 - Gráfico do resultado final para a métrica satisfação.

\section{Conclusões}

Os resultados obtidos com esse estudo visam apresentar uma nova vertente para avaliação de usabilidade de sistemas com objetivo de melhoria de produtividade das organizações. Dependendo do sistema analisado, esse resultado pode ser interpretado como retenção de clientes, aumento do lucro, satisfação dos empregados, aumento da produtividade dos funcionários, entre outros benefícios.

Nesse caso, a metodologia foi aplicada à intranet de uma grande empresa sobre uma amostra de vinte e sete empregados. A intranet é uma ferramenta corporativa com várias funcionalidades fundamentais para a empresa, a sua interface não pode ser um entrave na utilização de suas funções. O software deve ser fácil de aprender e de relembrar, não provocar erros e o usuário deve concluir suas tarefas com eficiência e eficácia. Todos esses fatores implicam na satisfação final do usuário para com o produto.

Após a análise dos resultados parciais dessa pesquisa, obteve-se uma avaliação da usabilidade da intranet da Eletrobrás, conforme apresentada a seguir:

Com relação às métricas Facilidade de Aprender e Facilidade de Relembrar, o resultado foi uma usabilidade Alta, porém com pouca confiabilidade nos dados por haver uma dispersão muito grande das opiniões dos usuários.

A métrica Controle de Erros foi avaliada com usabilidade Alta, mas foi que teve a pior confiabilidade, ou seja, também houve uma dispersão muito grande das opiniões dos usuários.

As métricas Eficiência, Eficácia e Satisfação obtiveram índices mais convergentes das opiniões dos usuários e, por conseqüência, uma confiança maior nos resultados que foram respectivamente Alta, Média e Alta usabilidade.

Os quesitos Controle de Erros e Eficácia foram os que apresentaram piores desempenhos no resultado final. Entrando no detalhe de cada uma dessas métricas, a pesquisa mostrou que com a melhoria de alguns detalhes, pode-se conseguir resultados melhores para esses critérios, e por conseqüência na usabilidade final. Esses resultados serão apresentados para a empresa pesquisada no intuito de melhorar o desempenho de sua intranet.

Uma ação essa que poderia melhorar muito o desempenho do uso dessas ferramentas corporativas seria a consulta dos usuários, nesse caso os funcionários, quando do desenvolvimento de ferramentas que afetam diretamente o seu trabalho. Os funcionários muitas vezes acabam tendo que usar os softwares do jeito que lhes são disponibilizados e pela própria correria e pressão do trabalho no dia-a-dia acabam por se adaptarem às interfaces dos sistemas.

Outra conclusão foi a de não existir uma avaliação única para usabilidade de um sistema. Na verdade existem vários critérios ou métricas que, em conjunto, acabam por representar a usabilidade do sistema com um todo. Um sistema pode ser fácil de aprender, mas apresentar muitos erros ou falhas que acarretam na perda de informações, por exemplo.

\section{Referências}

[1] BARRETO, Jorge M. Inteligência Artificial no Limiar do Século XXI. Florianópolis: Duplic, 2001.

[2] BRAGA, Mario J. F.; BARRETO, Jorge M.; MACHADO, Maria Augusta S. Conceitos da Matemática Nebulosa na Análise de Risco. Rio de Janeiro: Artes\& Rabiskus, 1995.

[3] CEZAR, Breno L., MACHADO, Maria Augusta S., OLIVEIRA JR, Hime A. Sistema de Apoio à Decisão na Concessão de Crédito Pessoal usando Lógica Fuzzy. Anais do Simpósio de Excelência em 
Gestão e Tecnologia (SEGET), Rio de Janeiro, 2006.

[4] CYBIS, Walter de Abreu. Engenharia de Usabilidade: Uma Abordagem Ergonômica. Florianópolis, Maio de 2003. Disponível em: $<$ http://www.unoescsmo.edu.br/poscomp/cybis/Apos tila_v51.pdf>. Acesso em: 24 mar. 2006.

[5] ELETROBRÁS. Site Institucional. Disponível em: http://www.eletrobras.gov.br. Acesso em: $02 \mathrm{fev}$ 2007.

[6] GRALLA, Preston. Como funcionam as intranets. São Paulo, Quark, 1996.

[7] ISO 9126-1. Engenharia de software - Qualidade de produto. Parte 1: Modelo de qualidade. NBR ISO/IEC 9126-1. Rio de Janeiro: ABNT, 2003.

[8] ISO 9241. Ergonomic requirements for office work with visual display terminals (VDTs). ISO 9241. Switzerland: ISO, 1998.

[9] KALAKOTA, Ravi; ROBINSON, Marcia. Ebusiness: Estratégias para Alcançar o Sucesso no Mundo Digital. Porto Alegre: Bookman, 2002.

[10] MATTAR, F.. Pesquisa de Marketing. São Paulo: Editora Atlas, 1997.

[11] NIELSEN, J. Usability Engineering. Boston, MA: Academic Press, 1993.

[12] O’BRIEN, James. Sistemas de Informação e as Decisões Gerenciais na Era da Internet. São Paulo: Saraiva, 2002.

[13] OLIVEIRA JR., Hime A., CALDEIRA, André M., MACHADO, Maria A. S., SOUZA, Reinaldo, TANSCHEIT, Ricardo. Inteligência Computacional Aplicada à Administração, Economia e Engenharia em Matlab. Rio de Janeiro, Thompson, 2007.

[14] PRESSMAN, R. S. Software Engineering - A Practioner's Approach, 3 ed., McGraw-Hill, 1992.

[15] QUESENBERY, W. What does usability mean: Looking beyond 'ease of use'. In: 48th Annual Conference Society for Technical Communication. Chicago, 2001.

[16] SANTOS, Rodrigo C.; MACHADO, Maria Augusta S. Development of a Methodology for Systems Usability Evaluation Using Fuzzy Logic Based on ISO. Anais do X Simpósio de Administração da Produção, Logística e Operações Internacionais (SIMPOI 2007). Rio de Janeiro, 2007.
[17] TURBAN, Efraim; KING, David. Comércio Eletrônico: Estratégia e Gestão. São Paulo: Prentice Hall, 2004.

[18] VERGARA, Sylvia C. Projetos e Relatórios de Pesquisa em Administração. 3 ed. São Paulo: Atlas, 2000. 